\title{
Digital Diet: A 21st century approach to understanding digital technologies and development
}

\author{
Amy Orben ${ }^{1,2}$ \\ ${ }^{1}$ MRC Cognition and Brain Sciences Unit, University of Cambridge \\ ${ }^{2}$ Emmanuel College, University of Cambridge
}

How does screen time affect child development?

Can adolescents cope with the increased time they spend on social media?

Will a digital childhood negatively impact later outcomes?

21 st century parents, policymakers and researchers routinely ask questions about how digital technologies might affect child and adolescent development. The popularity of these queries should not be surprising to us: digital technologies are changing what it means to communicate, work and learn. They are not just influencing our lives by reforming the jobs we do and the social systems we inhabit; they are influencing the lives of children and young people who inhabit a world increasingly shaped by these innovations. We urgently need timely and high-quality evidence about how these changes impact children and adolescents, to develop practical approaches that can ensure the best outcomes for our youngest generations, and society as a whole.

For years, the default psychological approach to addressing questions about technology and development was to try and establish evidence-based screen time guidelines, i.e. concrete amounts of time that children and adolescents are recommended to spend on digital technologies to avoid negative impacts (Davies et al., 2019). Researchers would search for a concrete numerical relationship between time spent on digital technologies (i.e. so-called screen time, see also Kaye et al., 2020) and various outcomes of interest such as well-being, self-esteem, depression or attention difficulties (for review see Odgers \& Jensen, 2020). The implicit assumption of this research was that there is an informative and simple numerical relationship connecting the time spent using digital technologies and developmental outcomes (also known as a dose-response relationship, see Orben et al., 2020).

I have come to believe that this collective search for a uni-

AO was supported by a College Research Fellowship from Emmanuel College, University of Cambridge and by the UK Economic and Social Research Council ES/T008709/1.

Correspondence concerning this article should be addressed to Amy Orben, MRC Cognition and Brain Sciences Unit, University of Cambridge. E-mail: aco35@cam.ac.uk tary numerical value linking screen time with developmental outcomes is futile, primarily because such a value does not exist.

For a long time, there has been a mistaken assumption that research and public policy approaches could treat time spent on digital devices as they have other chemical compounds studied for public health purposes. While it has been possible to create guidelines about how many "units" of alcohol one should drink, establishing evidence-based guidelines about how many minutes of social media a child should use is much more difficult (e.g. see Hawkes, 2019). Time spent on digital devices does not act like a chemical compound which, when ingested, leads to quantifiable changes in the body that are relatively uniform across populations. This mistaken assumption has made many research outputs conflicting and largely uninformative (Dienlin \& Johannes, 2020). The relationship between the use of digital technologies and developmental outcomes will depend on many factors including, but not limited to, the type of digital technology used, the technology user, the user's motivations, their socio-cultural context and their environment. Because digital technologies now mediate many aspects of societal life, they have developed into a diverse landscape that no single numerical estimate can adequately summarize or explain.

I intend to use this piece to introduce the digital diet approach: a metaphor that challenges how we currently research and reason about digital technologies. The digital diet approach draws on parallels to our established approach for understanding and reasoning about diet, something that has been recognized by multiple technology practitioners (Teixeira and Caio, 2019; Brewer, 2021) and researchers (Bavelier et al., 2011).

I acknowledge that questions about what we eat are not identical to questions about what digital technologies we use. As stated above, technology use is not a chemical compound that can be ingested and that has a common impact on the body. Research questions about technology use therefore often demand more complicated answers than research questions about food. An example of this additional complexity is how digital technologies can both be considered a passive medium used to gain access to content (e.g. I use social media to read polarized news), and an active medium that determines the sort of content accessed in the first place (e.g. social media algorithms make me see more polarized news). While 
in this piece I conflate both ways of conceptualizing digital technologies, and oversimplify digital technology use in the processes, in future we need to develop approaches that can adequately address such complexity.

Until such approaches are established, there are concrete parallels between the study of food and the study of technologies that can help hone our thinking about digital technologies' influence on development. For example, to understand diet we need to think about a) what is being eaten, b) the amount that is being eaten, c) different food groups, d) individual differences and e) population differences. Similar questions need to be raised when trying to understand digital diets. To detail this, I will address six conceptual starting-points of the metaphor below; each describes - in broad strokes - a different conceptual angle of the digital diet approach and how it diverges from current practices in the psychological sciences.

\section{Type}

We all would agree that not all foods are created equal: some are healthier or unhealthier than others. It should not come as a surprise that this is the same for digital technologies. Past work investigating the effects of time spent using digital technologies has, however, routinely overlooked such nuance (for examples see Orben \& Przybylski, 2019; Twenge et al., 2017). Our research practice needs to acknowledge that both the type of technology used and the type of content consumed are important determinants of the impact such use will have on children and young people. For example, 20 minutes of scrolling through abusive content on social media will have a different impact when compared to 20 minutes of reading on an iPad or 20 minutes of using the same iPad to join an exercise class.

\footnotetext{
Amount

This is pretty simple: how much you eat of what matters. The amount that is healthy to consume might differ when comparing various types of food or drink. For example, small amounts of certain products (e.g. alcohol in adults) can have relatively little effect, whereas larger quantities can incur larger impacts. Extreme amounts are often related to problematic outcomes. This is also true in the digital diet approach. Research has found that there might be a benefit for young people using different technologies for different amounts of time (Przybylski \& Weinstein, 2017). Further, the amount of use needs to be considered in context: one hour of additional gaming on a console will not have the same effect if applied to a teenager who regularly plays 30 minutes, compared to one who plays 8 hours a day.
}

\section{Balance}

A research field only studying food groups in isolation will be unable to fully understand diet. Holistic approaches that can establish the balance between different types of food, e.g. to locate specific deficiencies, are important contributions to the research literature. This is something still largely ignored by developmental research studying digital technology use (for perspective see Büchi, 2020). Most work either focuses on the use of specific devices or "screen time" as a whole (Meier $\&$ Reinecke, 2020). There needs to be additional holistic work available that studies not just one type of technology use, especially as digital devices are now being used side-byside to maximise and customise technological experiences (Ofcom, 2020; Doughty et al., 2012). Teenagers might talk to each other over FaceTime while watching a movie on their TV or iPad; they might also scroll through social media on their phone while doing their homework on a laptop. Studying technologies in combination is however very difficult, and might need either in depth tracking over multiple devices or mixedmethods approaches where individuals are shadowed over longer periods of time to document their technology usage. If we only stick with the status quo and study technologies in isolation, we might not be seeing the whole picture.

\section{Individuals}

Most of us can eat most foods without experiencing any clinical harm. However, some of us react very differently when consuming certain food types. This all comes down to individual differences. An extreme case of an individual difference is a severe food allergy. Those with severe allergies require different public policy approaches to minimise their exposure to certain foods and medical interventions to treat them if they become exposed. One could postulate that this is also relevant for digital diets. Certain experiences, cognitive approaches or environmental stimuli might heighten an individual's vulnerability to certain digital inputs, e.g. clinically depressed teenagers seeing self-harm content on social media sites (Odgers, 2018). Individual differences in sensitivity to digital diets might also be present in less severe forms: personality, life history, self-regulation ability and demographics could make some children and young people more sensitive to digital technologies than others. Further, age can greatly influence both the types of digital technologies used and how they affect the individual child or young person. We ultimately need to understand these differences to design scientific, policy and clinical approaches that enable all children and young people to thrive in a digital world. 
Utility

We further need to acknowledge the importance of studying digital device use in context, because the purposes that digital devices are used for might determine their impact. This reasoning is already established in our approach to diet. An energy bar during an ultra-marathon has a very strategic utility: to provide energy to a deficient system in a time of stress. In the technology space, this could be equivalent to an adolescent using social media to organise climate strikes, a toddler video-calling distant grandparents or a teenager creating a chatroom for friends during a COVID-19 lockdown. In all of these cases, digital technologies are being used to address a concrete need, and they address this need much better than other non-digital tools (Orben, Tomova, et al., 2020). The energy bar and the technological device, however, could have a very different impact if they were consumed for a different reason: for example because of boredom or absentmindedness. An appreciation of the various utilities of digital technology use might therefore be crucial for understanding how it affects development.

\section{Population}

Different populations around the world struggle with different dietary challenges: from poverty and malnutrition to obesity. While some populations need to curb consumption, others struggle to provide the basic level of nutrition that allows people to flourish. In the digital world certain parallels can be drawn concerning digital inequality. There are clear benefits gained by children and their families through equitable access to the digital world (e.g. in terms of education or healthcare, Livingstone \& Bulger, 2014). This could be in the context of the Global South where getting access to a mobile phone can allow you to use important financial services or the Global North where, in the COVID-19 pandemic, access to devices to engage in online healthcare appointments or school lessons became necessary. No access to digital devices can thus cause harm, just as overuse of digital devices can cause harm.

However, once access is established other questions arise, and the effects of inequality might reverse. In many countries in the Global North unhealthy food is now accessible and cheap, while fresh and healthy food is a luxury disadvantaged communities cannot afford. One could extrapolate this to the digital world, arguing that once basic digital access is established those at the greatest disadvantage might experience more technological harms than other populations. A truly global research perspective needs to define the populations studied and how this determines the research approach taken, keeping in mind that each approach might well not translate to other populations around the world.
Social media as carbohydrates: an example

In concluding our exploration of the digital diet approach, we might want to imagine each type of digital technology as a different type of food. For sake of an easy example, we might equate social media to carbohydrates. Carbohydrates come in many different forms: wholemeal pasta, white bread, cakes or potatoes. Social media also comes in many different forms, with many different types of content and design features. In everyday life many would understand the limitations of trying to find a universal link between eating a certain amount (e.g. 20g) of carbohydrates and well-being. Some might avoid carbohydrates to help control conditions like diabetes, while others might seek out a carbohydrates to support challenging physical tasks or exercise regimes. Without knowing the frequency of eating carbohydrates we would also not be able to say much: if we are studying a person eating $20 \mathrm{~g}$ of carbohydrates every 30 seconds we would need to come to a different conclusion than if we are studying a person eating $20 \mathrm{~g}$ of carbohydrates a day or week. As psychologists, we might also want to know about the motivations for eating carbohydrates. For example, does the person we are studying eat this food because they are hungry, lonely or because they cannot stop themselves? In all, we clearly need to acknowledge many different dimensions before coming to a conclusion about how the consumption of carbohydrates might affect an individual.

From a digital diet perspective, the same nuance is needed to start understanding how social media use affects individuals and their development. Are there specific groups of children and young people especially affected by their use of social media (just like diabetics or high endurance athletes)? Does the frequency and type of social media use influence these effects? Are psychological factors like motivations or utility important to consider? Acknowledging this complexity will be fundamental to successfully understanding how digital technologies influence development. Yet this metaphor also has its limits: technology use is not the same as eating food and comes with additional questions and complexities. We might risk glossing over important nuances if we solely take a digital diet perspective (e.g. as seen in the conversation about digital detox). This makes it important that the digital diet approach is just used as a stimulus for further nuanced and complex thinking, rather than a means to an end.

\section{What does the future hold?}

While the digital diet approach ultimately remains a metaphor, it makes abundantly clear that simple answers to questions about digital technologies and development might be far out of our reach - and that they might remain far out of reach for a long time. Indeed, research on diet remains fraught and uncertain even after decades of research. 
There is an inherent complexity in trying to understand, in general, how an individualized medium affects individuals when we know these impacts will be highly individualized themselves. The ever-changing technology landscape, where new technologies are developed at increasingly rapid speeds, provides additional challenge to this task. It might therefore be too ambitious to believe that in 10 years we will have a uniform understanding of the impacts of digital technologies on development. With help of better theoretical and statistical models (Orben et al., 2020), a more realistic aim might be that by then we will understand whether there are specific groups of technology users or specific designs of technologies that we should be concerned about. This could include examining whether those children who are already most vulnerable in terms of their cognitive approaches, clinical history or environment also are impacted by these vulnerabilities online, or investigating very specific types of technology use in isolation.

Taking a digital diet approach could allow developmental psychology to move away from a mistaken overgeneralisation: but it will mean tackling an inherently more complicated research task. Yet the stakes are high, and some of the most pressing developmental research questions of the 21 st century remain unanswered. I think we should rise to the challenge.

\section{References}

Bavelier, Daphne, C. Shawn Green, Doug Hyun Han, Perry F. Renshaw, Michael M. Merzenich, and Douglas A. Gentile. "Brains on Video Games". Nature Reviews Neuroscience 12, no. 12 (December 2011): 763-68. https://doi.org/10.1038/nrn3135.

Büchi, Moritz. "A Proto-Theory of Digital Well-Being”. Preprint. Open Science Framework, 8 September 2020. https://doi.org/10.31219/osf.io/k3e2j.

Brewer, Jocelyn. "Digital Nutrition". Digital Nutrition. Accessed 18 February 2021. https://jocelynbrewer. $\mathrm{com} /$.

Davies, S. C., Atherton, F., Calderwood, C., \& McBride, M. (2019). United Kingdom Chief Medical Officers' commentary on "Screen-based activities and children and young people's mental health and psychosocial wellbeing: A systematic map of reviews". Department of Health and Social Care.

Dienlin, T., \& Johannes, N. (2020). The impact of digital technology use on adolescent well-being. Dialogues in Clinical Neuroscience, 22(2), 135-142. https://doi.org/10.31887/DCNS.2020.22.2/tdienlin

Doughty, Mark, Duncan Rowland, and Shaun Lawson. "Who Is on Your Sofa?: TV Audience Communities and Second Screening Social Networks". In Proceedings of the 10th European Conference on Interactive TV and Video - EuroiTV'12, 79. Berlin, Germany: ACM Press, 2012. https://doi.org/10.1145/2325616. 2325635.

Hawkes, Nigel. "CMO Report Is Unable to Shed Light on Impact of Screen Time and Social Media on Children's Health.” BMJ 364 (8 February 2019): 1643. https://doi.org/10.1136/bmj.1643.

Kaye, L. K., Orben, A., Ellis, D. A., Hunter, S. C., \& Houghton, S. (2020). The Conceptual and Methodological Mayhem of "Screen-time" [Preprint]. Open Science Framework. https://doi.org/10.31219/osf.io/ u4hqn

Livingstone, S., \& Bulger, M. (2014). A Global Research Agenda for Children's Rights in the Digital Age. Journal of Children and Media, 8(4), 317-335. https: //doi.org/10.1080/17482798.2014.961496

Meier, A., \& Reinecke, L. (2020). Computer-Mediated Communication, Social Media, and Mental Health: A Conceptual and Empirical Meta-Review. Communication Research, 009365022095822. https: //doi.org/10.1177/0093650220958224 
Odgers, C. L. (2018). Smartphones are bad for some teens, not all. Nature, 554(7693), 432-434. https://doi.org/ 10.1038/d41586-018-02109-8

Odgers, C. L., \& Jensen, M. R. (2020). Annual Research Review: Adolescent mental health in the digital age: facts, fears, and future directions. Journal of Child Psychology and Psychiatry, 61(3), 336-348. https://doi.org/10.1111/jcpp.13190

Ofcom. (2020). Ofcom Children's Media Lives: Life in Lockdown. Ofcom.

Orben, A., \& Przybylski, A. K. (2019). The association between adolescent well-being and digital technology use. Nature Human Behaviour, 3(2), 173-182. https://doi.org/10.1038/s41562-018-0506-1

Orben, A., Tomova, L., \& Blakemore, S.-J. (2020). The effects of social deprivation on adolescent development and mental health. The Lancet Child \& Adolescent Health, 4(8), 634-640. https://doi.org/10.1016/ S2352-4642(20)30186-3

Orben, A., Weinstein, N., \& Przybylski, A. K. (2020). Only
Holistic and Iterative Change Will Fix Digital Technology Research. Psychological Inquiry, 31(3), 235-241. https://doi.org/10.1080/1047840X.2020. 1820221

Przybylski, A. K., \& Weinstein, N. (2017). A Large-Scale Test of the Goldilocks Hypothesis. Psychological Science, 28(2), 204-215. https://doi.org/10.1177/ 0956797616678438

Fabricio, Teixeira, and Braga Caio. "The World Needs a Tech Diet; Here Is How Designers Can Help". The world needs a tech diet; here is how designers can help, 26 May 2019. http://essays.uxdesign.cc/1408036/.

Twenge, J. M., Joiner, T. E., Rogers, M. L., \& Martin, G. N. (2017). Increases in Depressive Symptoms, SuicideRelated Outcomes, and Suicide Rates Among U.S. Adolescents After 2010 and Links to Increased New Media Screen Time. Clinical Psychological Science, 6(1), 3-17. https://doi.org/10.1177/ 2167702617723376 